\title{
Amyotrophic Lateral Sclerosis: a comparison of two staging systems in
}

\section{a population-based study}

${ }^{1}$ Diana Ferraro MD PhD, ${ }^{2}$ Dario Consonni MD, MPH, PhD, ${ }^{1}$ Nicola Fini MD, ${ }^{1}$ Antonio Fasano MD, ${ }^{3}$ Cinzia Del Giovane PhD, ${ }^{4}$ ERRALS Group, and ${ }^{1}$ Jessica Mandrioli MD.

(1) Department of Biomedical Metabolic and Neurosciences, University of Modena and Reggio Emilia, Nuovo Ospedale Civile S. Agostino Estense, Modena, Italy

(2) Epidemiology Unit, Fondazione IRCCS Ca' Granda - Ospedale Maggiore Policlinico, Milan, Italy

(3) Department of Diagnostic and Clinical Medicine and Public Health, University of Modena and Reggio Emilia, Modena, Italy

(4) Emilia-Romagna Registry for ALS Group

\section{Corresponding author:}

Jessica Mandrioli, MD

Department of Neurosciences, Nuovo Ospedale Civile S. Agostino Estense

Via Pietro Giardini n. 1355

41126 Modena, Italy

Tel. 00390593961640 , Fax 00390593963775

E-Mail j.mandrioli@ausl.mo.it

Search terms: amyotrophic lateral sclerosis, staging, prognosis, population-based study

Word count: 3180

Running title: Staging Systems in ALS

\section{Author Disclosures}

Jessica Mandrioli has received research support from Regione Emilia Romagna (Emilia Romagna Registry for Amyotrophic Lateral Sclerosis). 


\section{ABSTRACT}

Objective: to assess advantages and drawbacks of two recently proposed staging systems for Amyotrophic Lateral Sclerosis (ALS) - King's College and MITOS staging system - in an incident, population-based cohort of ALS patients. Methods: Since 2009, a prospective registry records all incident cases of ALS in the Emilia Romagna region, Italy. For each patient, detailed clinical information, including the ALS functional rating scale (ALSFRS-R) score, is collected at each follow-up.

Results: Our study on 545 incident cases confirmed that King's College stages occur at predictable times throughout the disease course (at 40, 60 and $80 \%$ of the disease course) while MITOS stages are skewed towards later stages (35, 67, 79, 100 and 104\% of the disease course). In King's College system there is a decrease in survival and an increase in deaths with escalating stages, while in the MITOS system survival curves pertaining to intermediate stages overlap and the number of deaths is fairly homogeneous throughout most stages.

Conclusions: King's College staging system has a higher homogeneity (i.e. smaller differences in survival among patients in the same stage),and a higher discriminatory ability (i.e. greater differences in survival among patients in different stages), being more suitable for individualized prognosis and for measuring efficacy of therapeutic interventions. 


\section{INTRODUCTION}

Amyotrophic lateral sclerosis (ALS) is a neurodegenerative disorder characterized by progressive disability [1] and great inter-individual variability, making prognosis still a challenge. Although several prognostic models exist, based on clinical/demographic factors at diagnosis for prediction of survival, they do not include milestones, which are necessary for staging criteria [2]. A reliable staging system would be of great importance as it could help predicting prognosis, it would allow personalized counselling, and it would be useful for clinical trial conduction and resources allocation. The most widely used functional scale in ALS [the ALS Functional Rating Scale- Revised (ALSFRS-R)], has relevant intrinsic limitations: it is multidimensional, representing the sum of mean scores of three different domains, thus not satisfying rigorous measurement standards $[3,4]$.

To address this urgent unmet need, recently, two staging systems have been developed [5, 6], based on simple clinical milestones marking the course of the disease.

The first [5] staging system, hereafter called "King's College staging system", considers the number of involved regions for the first three stages and need for gastrostomy and for non-invasive ventilation for the subsequent stage (4A and $4 \mathrm{~B}$, respectively).

The second staging system (namely MITOS system)[6] is based on the loss of independent functions in four key domains of the ALSFRS-R: the sum of the lost functions determines the stage. Both systems have only been tested separately in clinical trials/referral-centre populations. To our knowledge, studies on the validity and applicability of comparable models and systems for functional status in the general ALS population are lacking.

\section{MATERIALS AND METHODS}

\section{Patients}

Since 2009, a prospective registry (ERRALS)[8] records all incident cases of ALS in the Emilia Romagna region, Italy. For each patient, detailed clinical information, including the ALSFRS-R score [7] is collected at each follow-up.

\section{Protocol approval}

All patients signed an informed consent permitting the treating neurologist to record their data in the registry, which was approved by the ethics committees of the coordinating centre and of the nine Provinces of Emilia Romagna.

\section{Staging systems}

King's College staging system [5] considers the number of involved regions for the first three stages (but distinguishing time of diagnosis -stage 2A-from involvement of the second region-stage 2B-) and need for gastrostomy and for non-invasive ventilation for stages 4A and 4B, respectively. Stage 5 corresponds to death. The stages were well distributed during the disease course, without reversion to earlier stages, with good correlation with 
ALSFRS-R scores [8]. After having been tested on a prevalent, centre-based cohort, this system has been applied to 725 patients enrolled in two clinical trials (LICALS and MITO) $[9,10]$, calculating stages by starting from ALSFRS-R scores obtaining an estimated stage which correlated about $92 \%$ with actual clinical stage [11]. Considering that diagnosis may be reached virtually at any point of the disease course, the authors merged stages $2 \mathrm{~A}$ and $2 \mathrm{~B}$ into stage 2 , that was simply reached with involvement of a second region. In the present study, King's College stages were calculated considering the loss of at least 1 point in any item of the ALSFRS-R referring to a certain body region. The MITOS system is based on the loss of independent functions in four key domains of the ALSFRS-R; the sum of the lost functions determined the stage of ALS-MITOS, including 6 stages from 0 (absence of functional loss in any domain) to 4 (loss of function in 4 domains); Stage 5 is represented by death. The authors tested MITOS system using data obtained from two clinical trials (LITALS and QOC study) [12, 13]; then in a cohort of 200 ALS patients randomized in the EPOS study [14]. MITOS stages were calculated considering when loss of function, as defined by Chiò and colleagues [6], occurred at ALSFRS-R.

\section{Statistical methods}

Chi-square test, was used to explore differences between groups for categorical data, equality of medians and KruskalWallis tests for continuous data.

Median time to each clinical stage was standardized by dividing the time from onset to each clinical milestone by the disease duration and multiplying by 100, using only information from patients who had died.

Equality of medians test was used to explore differences between median times from onset to each stage, for each of the staging systems.

Clinical stage was treated as time-dependent variable, i.e., for each patient we calculated the person-time at risk during each stage. Kaplan-Meier survival curves followed by log-rank test were used to evaluate survival during each clinical stage of both staging systems.

For comparison of staging systems we used the Cox regression model to calculate the log likelihood in order to determine homogeneity and the Cochran-Armitage test for trend to measure the discriminatory ability of each staging system.

Data were analyzed using Stata 11 (StataCorp, Texas, USA).

\section{RESULTS}

\section{Patient characteristics}

We analyzed 545 consecutive newly diagnosed ALS from $1^{\text {st }}$ January 2009 to $31^{\text {st }}$ December 2013 in Emilia Romagna Region of Italy. Patient characteristics are shown in Table 1. 


\section{Standardized median time to each milestone: comparison of staging systems}

Figure 1 shows standardized median times (SMT) to stages 1, 2, 3, 4a, $4 \mathrm{~b}$ and 5 (death), where 0 is onset of disease and 100 is death, according to King's College classification.

Figure 2 shows SMT to stages $0,1,2,3,4$ and 5 (death) according to the MITOS staging system.

In both cases, we only used information from patients who had reached the end of the disease course, having died.

Table 2 shows median and SMT from onset to each milestone and comparison of medians between pairs of consecutive stages.

\section{Number/proportion of deaths throughout stages}

In King's College staging system there is a steady increase in the number and in the proportion of patients who die throughout stages 1 to $4 \mathrm{a} / \mathrm{b}$, when considering the last recorded milestone (stage 1: 14/65-22\%; stage 2: 24/67-36\%; stage 3: 53/118-45\%; stage 4a: 33/55-60\%; stage 4b: 148/239-62\%). Ninety percent of tracheostomized patients undergo tracheostomy during stage 4 of King's College stages (4a: $28 \%$ and $4 \mathrm{~b}: 62 \%$ ).

In the MITOS system the number and/or proportions of deaths are fairly homogeneous throughout stages 2-4 (stage 0: 58/174-33\%; stage 1: 69/149-46\%; stage 2: 63/92-69\%; stage 3: 39/62-63\%; stage 4:43/67-64\%). The percentage of patients undergoing tracheostomy was evenly spread out throughout stages $0-3(0: 21.5 \%, 1: 21.5 \%, 2: 23 \%, 3: 25 \%, 4$ : $9 \%)$.

\section{Survival curves: comparison of staging systems}

Figures $3 \mathrm{a}$ and $3 \mathrm{~b}$ depict Kaplan Meier curves for time to death/last observation during each clinical stage for the King's College (Fig. 3a) and for the MITOS (Fig. 3b) staging system, followed by log-rank test and Cox analyses between pairs of subsequent stages.

\section{Comparison of prognostic stratification of staging systems}

Table 3 shows the two staging systems' discriminatory ability and homogeneity for prediction of time to death/last observation. King's College system shows both a higher homogeneity within stages and a higher linear trend.

\section{DISCUSSION}

The present study was aimed at assessing advantages and drawbacks of two recently proposed staging systems for ALS patients when applied in an incident, population-based, cohort.

\section{Standardized median time to each milestone and cohort effects on timings}

According to King's College staging system, standardized median time from onset to weakness in a second region, weakness in a third region and the need for gastrostomy or respiratory support (stages $2,3,4 \mathrm{a}$ or $4 \mathrm{~b}$, respectively) occur 
at distinct times, corresponding approximately to 40,60 and $80 \%$ of the disease course, respectively. Our data is very similar to theirs, with above-said milestones occurring at $42,58,81$ and $73 \%$ of the disease course.

Stages 0-4 of the MITOS staging system are reached after a SMT of 35, 67, 79, 100 and 104\% of the disease course in our cohort.

Overall, King's College milestones are more evenly spaced out throughout the disease course while the MITOS milestones are skewed towards later stages of the disease, with SMT to stages 3 and 4 being equal to or longer than median time to death. This is probably due to the fact that each stage is reached only once a complete loss of function has occurred and this occurs towards the end of the disease course.

Median time spent in each stage varies from 2-8 months for King's College, similarly to data from two large phase 3 clinical trials assessed by Balendra et al [8], in which median duration of transition time from one stage to another varied from 3 to 7 months. For MITOS systems the median duration of stages 0-2 ranged from 3 to 9 months while the median time from onset to stages 3, 4 and 5 overlapped.

As for subgroups of patients, median time to weakness in a third region is anticipated in patients with a bulbar onset both in our cohort (SMT: 43) and in King's College cohort (SMT: 45). This is consistent with the worse prognosis of the bulbar forms.

\section{Survival curves during each stage}

In King's College system there is a decrease in survival from the earliest to the most advanced stages when considering time to death/last observation during each stage and survival curves are all significantly distinct from one another. In the MITOS staging system survival during stage 2 does not differ significantly from survival during stage 3 and, paradoxically, there seems to be an inversion of the curves with patients dying sooner during stage 2 of the disease as opposed to those that have reached stage 3 (Figure 3b). Although not specifically addressed, data in the study by Chiò et al [6] is in line with our observations: the probability of transition from stage 2 to death and from stage 3 to death at one year is 33\% for both stages in the Quality of Care in ALS study [13], while in the LiTALS study [9], the probability even decreases from 29 to $25 \%$ for stage 2 and 3, respectively. We hypothesize that this may be due to the fact that patients who do not die after the complete loss of function in two domains, may belong to a group of "long survivors".

\section{Tracheostomized patients}

Eighty-two (15\%) patients underwent tracheostomy; of these, 47 (57\%) had died by the end of the follow-up. In our incident cohort, $90 \%$ of patients underwent tracheostomy during the last King's College stage while according to the MITOS system, the percentage of tracheostomies were similar throughout stages 0-3. 
Since without tracheostomy these patients would have died, it is not clear whether they should have been considered as patients who have reached the end of the disease course or not, based on the two considered staging systems. Neither of them addresses specifically this question, apparently considering tracheostomy as a region involvement (King's college Staging system) or a loss of function (MITOS system) whereas tracheostomy influences the global disease course as it remove the most frequent cause of death in patients suffering from ALS.

\section{The issue of dementia}

Neither King's College nor MITOS staging system included patients with dementia. In our cohort, the inclusion of patients with dementia anticipates median time to weakness in a third region using King's College staging system (milestones reached at 46, 54, 88 and $79 \%$ of the disease course) whereas the presence of dementia did not influence MITOS staging system (milestones reached at 67,81,102, and 110\% of the disease course). Since 15\% patients with ALS have concomitant fronto-temporal dementia [15], a staging system used in clinical practice should take cognitive involvement into account, and this issue may be the object of further studies.

\section{Strengths and Limits}

Strength of the present study is its population-based, real-life setting with inclusion of patients with dementia and a long follow-up.

A limit of the study is that data on time to clinical milestones is incomplete in some patients, especially in the more advanced stages. Furthermore, time to clinical stage was not collected prospectively, but was estimated retrospectively from ALSFRS-R.

\section{Conclusions}

In the present population, King's College milestones appear to be more evenly spaced out throughout the disease course and to be comparable to those described by Roche et al [5], while the MITOS milestones are skewed towards later stages of the disease.

Survival decreases steadily with escalating stages and survival curves are all distinct from one another in King's College staging system while in the MITOS system, survival curves during stages 2 and 3 do not significantly differ from one another.

Escalating stages are accompanied by an increasing number and proportion of deaths in King's College system while they are homogeneously spread out throughout stages 2-4 of the MITOS system.

King's College staging system has a higher homogeneity, i.e. small differences in survival among patients in the same stage within each system and a higher discriminatory ability, i.e. greater differences in survival among patients in different stages within each system compared to the MITOS system, suggesting a higher prognostic competency for the 
King's College staging system, especially for individual prognosis and for clinical trials use as an outcome measure.

However, the MITOS system, based on the complete loss of function in different domains, may be more useful for estimating health costs and resource allocations. [6].

Neither system specifically addresses patients with dementia and the step of tracheostomy and its importance during the disease course; further studies should deal with these issues.

\section{Figures titles and legends}

\section{Figure 1}

Title: Standardized median times to each milestone according to King's College staging system

Legend: Figure 1 showing SMT to involvement of one (stage 1), two (stage 2) and three (stage 3) CNS regions

(upper/lower limbs, diaphragmatic or bulbar), and to need for gastrostomy (stage 4a) or respiratory support (stage 4b).

\section{Figure 2}

Title: Standardized median times to each milestone according to the MITOS staging system

Legend: Figure 2 showing SMT to functional involvement in one domain (stage 0) and complete loss of function in one (stage 1), two (stage 2), three (stage 3) and four (stage 4) different domains (walking/self-care, swallowing, communicating, breathing).

\section{Figure 3a}

Title: Time to death/last observation during each stage: King's College staging system

Legend: Log-rank test and Cox analysis

Stage 1 vs 2: $\mathbf{p = 0 . 0 0 1 8}$ (HR: 2.7 [CI95: 1.4-5.3])

Stage 2 vs 3: $\mathbf{p = 0 . 0 0 2 6}$ (HR: 2 [CI95: 1.3-3.4])

Stage 3 vs 4a: $\mathbf{p = 0 . 0 0 5 8}$ (HR: $1.8[$ CI95: 1.2-2.8])

Stage 4a vs 4b: $\mathbf{p}<\mathbf{0 . 0 0 0 1}$ (HR: 2.8[CI95: 1.9-4.2])

\section{Figure 3b}

Title: Time to death/last observation during each stage: MITOS staging system

Legend: Log-rank test and Cox analysis

Stage 0 vs 1: $\mathbf{p}<\mathbf{0 . 0 0 0 1}$ (HR: 3 [CI95: 2.1-4,4])

Stage 1 vs 2: $\mathbf{p}<\mathbf{0 . 0 0 0 1}$ (HR: 2.4 [CI95: 1.7.-3.3])

Stage 2 vs $3: \mathrm{p}=0.2$

Stage 3 vs 4: $\mathbf{p = 0 . 0 0 0 8}$ (HR: 2.2[CI95: 1.4-3,5]) 


\section{References}

1. Kiernan MC, Vucic S, Cheah BC, et al. Amyotrophic lateral sclerosis. Lancet. 2011;377(9769):942-955.

2. Creemers H, Grupstra H, Beelen A. Prognostic factors for the course of functional status of patients with ALS : a systematic review. Journal of Neurology. 2015; 262:1407-1423.

3. Franchignoni F, Mandrioli J, Giordano A, Ferro S, Group E. A further Rasch study confirms that ALSFRS-R does not conform to fundamental measurement requirements. Amyotrophic Lateral Sclerosis 2015;6(5-6):331337

4. Franchignoni F, Mora G, Giordano A, Volanti P, Chiò A. Evidence of multidimensionality in the ALSFRS-R Scale : a critical appraisal on its measurement properties using Rasch analysis. Journal of Neurology Neurosurgery and Psychiatry 2013;84:1340-1345

5. Roche JC, Rojas-garcia R, Scott KM, et al. A proposed staging system for amyotrophic lateral sclerosis. Brain 2012; 135:847-852.

6. Chiò A, Hammond ER, Mora G, Bonito V, Filippini G. Development and evaluation of a clinical staging system for amyotrophic lateral sclerosis. Journal of Neurology Neurosurgery and Psychiatry 2015:38-44.

7. Cedarbaum JM, Stambler N, Malta E, et al. The ALSFRS-R: a revised ALS functional rating scale that incorporates assessments of respiratory function. BDNF ALS Study Group (Phase III). Journal of Neuroligical Sciences 1999;169(1-2):13-21.

8. Balendra R, Jones A, Jivraj $\mathrm{N}$, et al. Use of clinical staging in amyotrophic lateral sclerosis for phase 3 clinical trials. Journal of Neurology Neurosurgery and Psychiatry 2015;86(1):45-49.

9. Lenglet T, Lacomblez L, Abitbol JL, et al. A phase II-III trial of olesoxime in subjects with amyotrophic lateral sclerosis. European Journal of Neurology 2014;21(3):529-536.

10. Morrison KE, Dhariwal S, Hornabrook R, et al. Lithium in patients with amyotrophic lateral sclerosis (LiCALS): a phase 3 multicentre, randomised, double-blind, placebo-controlled trial. Lancet Neurology 2013;12(4):339-345.

11. Balendra R, Jones A, Jivraj N, et al. Estimating clinical stage of amyotrophic lateral sclerosis from the ALS Functional Rating Scale. Amyotrophic Lateral Sclerosis 2014;15(3-4):279-284.

12. Chiò A, Borghero G, Calvo A, et al. Lithium carbonate in amyotrophic lateral sclerosis: lack of efficacy in a dose-finding trial. Neurology 2010;75(7):619-625.

13. Filippini G, Bonito V, Chio A, Mora G, D’Alessandro R, Salvi F, Schoenhuber R, Cavalletti G, Galli A, Savettieri G, Testa D, Beghi E S V. Quality of life in patients with amyotrophic lateral sclerosis: the QuaC-ALS study database. Journal of Neurology 2003;250(S2):23.

14. Lauria G, Dalla Bella E, Antonini G, et al. Erythropoietin in amyotrophic lateral sclerosis: a multicentre, randomised, double blind, placebo controlled, phase III study. Journal of Neurology Neurosurgery and Psychiatry 2015;86(8):879-886.

15. Lomen-Hoerth C, Anderson T, Miller B. The overlap of amyotrophic lateral sclerosis and frontotemporal dementia. Neurology 2002;59:1077-1079. 


\section{Author Disclosures and Funding}

Jessica Mandrioli has received research support from Regione Emilia Romagna (Emilia Romagna Registry for Amyotrophic Lateral Sclerosis).

\section{Acknowledgements}

The ERRALS group (from Emilia Romagna Region, Italy) :

Project coordinator: J. Mandrioli, MD.

Collaborating centers: Department of Neuroscience, St. Agostino Estense Hospital, Modena (J. Mandrioli, N. Fini, A. Fasano, P. Nichelli); Department of Neurology, Bufalini Hospital, Cesena (S. Biguzzi, Y. Handouk, E. Venturini, M.G. Passarin); Department of Neurology, Forlì Hospital, Forlì (C. Guidi, W. Neri); Department of Neurology, St. Anna Hospital, Ferrara (E. Sette, V. Tugnoli, M.R. Tola); Department of Neurology, G. Da Saliceto Hospital, Piacenza (E. Terlizzi, D. Guidetti); Department of Neurology, Infermi Hospital, Rimini (M. Currò Dossi, M. Pasquinelli, J. Andruccioli, A. Ravasio); Department of Neurology, Faenza and Ravenna Hospital, Ravenna (M. Casmiro,F. Rasi); Department of Neurology and IRCCS Istituto delle Scienze Neurologiche di Bologna, Bellaria Hospital, Bologna (F. Salvi, I. Bartolomei, R. Michelucci); Department of Biomedical and Neuromotor Science and IRCCS Istituto delle Scienze Neurologiche di Bologna, University of Bologna, Bologna (P. Avoni, S. De Pasqua, R. Liguori); Department of Neurology, IRCCS Arcispedale Santa Maria Nuova, Reggio Emilia (R. Rizzi, E. Canali, N. Marcello); Department of Neuroscience, University of Parma, Parma, Italy (A. Grassi, L. Delay, V. Pietrini); Department of Clinical and Experimental Medicine, Respiratory Disease and Lung Function Unit, University of Parma, Parma (M. Aiello); Department of Neurology, Fidenza Hospital, Parma (D. Medici, E. Chierici, E. Montanari); Department of Neurology, Carpi Hospital, Modena (M. Santangelo, S. Amidei); Department of Neuroscience, University of Ferrara, Ferrara (I. Casetta, E. Groppo, E. Granieri); Department of Neurology, Imola Hospital, Bologna (P. De Massis, V. Mussuto); Department of Neurology and IRCCS Istituto delle Scienze Neurologiche di Bologna, Maggiore Hospital, Bologna (A. Borghi, A. Gabellini, T. Sacquegna); Department of Neuroscience, St. Orsola-Malpighi University Hospital, Bologna (R. Rinaldi, F. Cirignotta) Department of Hospital Services, Emilia Romagna Regional Health Authority, Bologna (S. Ferro); Neuroepidemiology Unit, IRCCS Istituto delle Scienze Neurologiche di Bologna, Bologna (R. D’Alessandro). 